\title{
Akademie Online startet ins Programmjahr 2019
}

\section{Akademie} Radiologie

AKADEMIE

FÜR FORT- UND WEITERBILDUNG IN DER RADIOLOGIE

in der Deutschen Röntgengesellschaft e.V.

Auf alle Teilnehmer der Kursreihe „Akademie Online“ wartet auch 2019 wieder ein vielfältiges und umfassendes Programm: Vom Lungenkrebsscreening im CT über die zerebrale Atrophie bis zur neuen Strahlenschutzgesetzgebung und was man in der Praxis dafür tun muss - das „Einschalten“ an jedem zweiten Dienstagabend lohnt sich! Den neuen Kursplan finden Sie ab sofort in unserem Online-Kalender unter www.drgakademie.de oder am Ende dieses Beitrags.

37 Kurse auf einen Streich buchen Sie mit dem günstigen Akademie-Online-Jahresabo - 25 Kurse für Ärztinnen und Ärzte sowie 12 Kurse, die speziell auf die Interessen für MTRA zugeschnitten sind. Das Jahresabo gilt jeweils für ein Kalenderjahr, d. h. bis zum 31.12. eines jeden Jahres. Für
DRG- und VMTB-Mitglieder gibt es das Jahresabo zum halben Preis!

Auch wenn Sie noch unentschlossen sind, können Sie Akademie Online jederzeit kostenfrei testen. Wählen Sie in unserem Kalender einfach eine der nächsten Veranstaltung aus und fordern Sie einen $\mathrm{Zu}$ gangslink unter Angabe der Option „kostenloser Testzugang“ an.

Alle Infos und die Möglichkeit zur Anmeldung finden Sie unter

www.drgakademie.de >Akademie Online.

\begin{tabular}{|c|c|c|}
\hline 08.01 .2019 & Pankreas & Prof. Dr. Johannes Weßling, Münster \\
\hline 22.01 .2019 & Labrumdiagnostik an Hüfte und Schulter & Prof. Dr. Marco Zanetti, Zürich \\
\hline 05.02 .2019 & MR-Neurografie & Prof. Dr. Mirko Pham, Würzburg \\
\hline 19.02.2019 & Koronarinfarkt CT & Prof. Dr. Konstantin Nikolaou, Tübingen \\
\hline 12.03 .2019 & Kontrastmittel im CT und MRT - zwischen Mythos und Wahrheit & Prof. Dr. Michael Uder, Erlangen \\
\hline 26.03.2019 & Lungenkrebsscreening im CT: Ist die Zeit reif dafür? & Prof. Dr. Stefan Diederich, Düsseldorf \\
\hline 09.04 .2019 & Bildgebung bei Hirntumoren & Prof. Dr. Elke Hattingen, Frankfurt \\
\hline 30.04.2019 & Neue Strahlenschutzgesetzgebung und was man in der Praxis tun muss & Prof. Dr. Dr. Reinhard Loose, Nürnberg \\
\hline 07.05 .2019 & Überlastungssyndrome im Sport & PD Dr. Marc Regier, München \\
\hline 21.05.2019 & Felsenbein-CT: diese Befunde muss ich kennen & Prof. Dr. Martin G. Mack, München \\
\hline 04.06 .2019 & Bildgebung der Finger und Ringbänder & Dr. Thomas Bayer, Erlangen \\
\hline 18.06.2019 & Zerebrale Atrophie - Grenzen des Normalen und Anfänge des Pathologischen & Prof. Dr. Sönke Langner, Rostock \\
\hline 02.07 .2019 & PET/CT: Das kleine und große $1 \times 1$ für Radiologen & PD Dr. Lale Umutlu, Essen \\
\hline 16.07.2019 & Differenzialdiagnose von Milzläsionen & Dr. Gernot Böhm, Linz \\
\hline 30.07 .2019 & Bildgebung der Leber mit leberspezifischen Kontrastmitteln & PD Dr. Martin Maurer, Bern \\
\hline 13.08.2019 & Hydrozephalus - mehr als nur weite Ventrikel & Prof. Dr. Stefan Hähnel, Heidelberg \\
\hline 27.08.2019 & MRT Kiefergelenk & PD Dr. Wolfgang Wüst, Erlangen \\
\hline 10.09.2019 & Knoten in der Brust - und nun? & Prof. Dr. Markus Müller-Schimpfle, Frankfurt a.M. \\
\hline 24.09.2019 & Systematische Befundung des Rektumkarzinoms & $\begin{array}{l}\text { Prof. Dr. Arnd-O. Schäfer (Leipzig) und Prof. Dr. Wieland } \\
\text { Sommer (München) }\end{array}$ \\
\hline 08.10 .2019 & Tumoren rund um die Wirbelsäule - Diagnostik und Differentialdiagnose & Prof. Dr. Tobias Struffert, Giessen \\
\hline 22.10 .2019 & $\begin{array}{l}\text { Wirbelsäule: Klassifikationen von Bandscheibenläsionen - Entzündungen } \\
\text { vs degenerative Veränderungen }\end{array}$ & Prof. Dr. Rolf Janka, Erlangen \\
\hline 05.11 .2019 & $\begin{array}{l}\text { Differenzialdiagnostik zystischer Lungenläsionen - ein häufiger } \\
\text { Zufallsbefund in der CT-Routinediagnostik }\end{array}$ & Dr. Beate Rehbock, Berlin \\
\hline 19.11.2019 & Frakturdiagnostik im Kindesalter & Prof. Dr. Markus Uhl, Freiburg \\
\hline 03.12 .2019 & Update MS-Bildgebung - neue Diagnosekriterien und Differentialdiagnostik & Prof. Dr. Arnd Dörfler, Erlangen \\
\hline 17.12 .2019 & Re-Staging unter Chemo- und Immuntherapie & Prof. Dr. Anno Graser, München \\
\hline
\end{tabular}

Bitte beachten Sie, dass sich auch kurzfristig noch Änderungen ergeben können! Das aktuelle Programm entnehmen Sie bitte unserem Online-Kalender unter www.drgakademie.de >Akademie Online > Kalender. 